\title{
Complete mitochondrial genomes of the 'intermediate form' of Fasciola and Fasciola gigantica, and their comparison with F. hepatica
}

Guo-Hua Liu ${ }^{1,3}$, Robin B Gasser ${ }^{2 *}$, Neil D Young ${ }^{2}$, Hui-Qun Song ${ }^{1}$, Lin Ai ${ }^{4}$ and Xing-Quan Zhu ${ }^{1,3^{*}}$

\begin{abstract}
Background: Fascioliasis is an important and neglected disease of humans and other mammals, caused by trematodes of the genus Fasciola. Fasciola hepatica and F. gigantica are valid species that infect humans and animals, but the specific status of Fasciola sp. ('intermediate form') is unclear.
\end{abstract}

Methods: Single specimens inferred to represent Fasciola sp. ('intermediate form'; Heilongjiang) and F. gigantica (Guangxi) from China were genetically identified and characterized using PCR-based sequencing of the first and second internal transcribed spacer regions of nuclear ribosomal DNA. The complete mitochondrial (mt) genomes of these representative specimens were then sequenced. The relationships of these specimens with selected members of the Trematoda were assessed by phylogenetic analysis of concatenated amino acid sequence datasets by Bayesian inference $(\mathrm{Bl})$.

Results: The complete mt genomes of representatives of Fasciola sp. and F. gigantica were 14,453 bp and 14,478 bp in size, respectively. Both mt genomes contain 12 protein-coding genes, 22 transfer RNA genes and two ribosomal RNA genes, but lack an atp8 gene. All protein-coding genes are transcribed in the same direction, and the gene order in both $\mathrm{mt}$ genomes is the same as that published for $F$. hepatica. Phylogenetic analysis of the concatenated amino acid sequence data for all 12 protein-coding genes showed that the specimen of Fasciola sp. was more closely related to F. gigantica than to F. hepatica.

Conclusions: The mt genomes characterized here provide a rich source of markers, which can be used in combination with nuclear markers and imaging techniques, for future comparative studies of the biology of Fasciola sp. from China and other countries.

Keywords: Liver fluke, Fasciola spp, Mitochondrial genome, Phylogenetic analysis

\section{Background}

Food-borne trematodiases are an important group of neglected parasitic diseases. More than 750 million people are at risk of such trematodiases globally [1,2]. Fascioliasis is caused by liver flukes of the genus Fasciola, and has a significant adverse impact on both human and animal health worldwide [3]. Human fascioliasis is caused by the

\footnotetext{
*Correspondence: robinbg@unimelb.edu.au; xingquanzhu1@hotmail.com 'State Key Laboratory of Veterinary Etiological Biology, Key Laboratory of Veterinary Parasitology of Gansu Province, Lanzhou Veterinary Research Institute, Chinese Academy of Agricultural Sciences, Lanzhou, Gansu Province, PR China

${ }^{2}$ Faculty of Veterinary Science, The University of Melbourne, Parkville, Victoria, Australia

Full list of author information is available at the end of the article
}

ingestion of freshwater plants or water contaminated with metacercariae of Fasciola [4]. It is estimated that millions of people are infected worldwide, and more than 180 million people are at risk of this disease worldwide [5]. To date, no vaccine is available to prevent fascioliasis. Fortunately, this disease can be treated effectively using triclabendazole [6], but there are indications of resistance developing against this compound [7].

The Fasciolidae is a family of flatworms and includes the genus Fasciola. Both F. hepatica and F. gigantica, which commonly infect livestock animals and humans (as definitive hosts), are recognized as valid species [8]. The accurate identification of species and genetic variants is relevant in relation to studying their biology, epidemiology 
and ecology, and also has applied implications for the diagnosis of infections. Usually, morphological features, such as body shape and perimeter as well as length/ width ratio, are used to identify adult worms of Fasciola [9]. However, such phenotypic criteria are unreliable for specific identification and differentiation, because of considerable variation and/or overlap in measurements between $F$. hepatica and F. gigantica [10].

Due to these constraints, various molecular methods have been used for the specific identification of Fasciola species and their differentiation [5]. For instance, PCRbased techniques using genetic markers in nuclear ribosomal (r) and mitochondrial (mt) DNAs have been widely used [11-13]. The sequences of the first and second internal transcribed spacers (ITS- 1 and ITS-2 = ITS) of nuclear rDNA have been particularly useful for the specific identification of $F$. hepatica and F. gigantica, based on a consistent level of sequence difference $(1.2 \%$ in ITS-1 and $1.7 \%$ in ITS-2) between them and much less variation within each species $[11,14]$. Nonetheless, studies in various countries, including China [5], Iran [15], Japan [16], Korea [14], Spain [17] and Tunisia [18], have shown that some adult specimens of Fasciola sp., which are morphologically similar to $F$. gigantica [10], are characterized by multiple sequence types (or "alleles") of ITS-1 and/or ITS-2, reflected in a mix between those of $F$. hepatica and F. gigantica [11,12]. Some authors [19-21] have suggested that such specimens (sometimes called intermediate forms') represent hybrids of F. hepatica and F. gigantica.

In the present study, we undertook an independent, genetic comparison of Fasciola sp. (i.e. 'intermediate form') and F. gigantica with F. hepatica. To do this, we characterized the mt genomes of individual specimens of Fasciola sp. and F. gigantica whose identity was defined based on their ITS-1 and/or ITS-2 sequences, and assessed their relationships by comparison with $F$. hepatica and various other trematodes using complete, inferred mt amino acid sequence data sets.

\section{Methods}

\section{Ethics statement}

This study was approved by the Animal Ethics Committee of Lanzhou Veterinary Research Institute, Chinese Academy of Agricultural Sciences (Permit code. LVRIAEC2012006). Adult specimens of Fasciola were collected from bovids, in accordance with the Animal Ethics Procedures and Guidelines of the People's Republic of China.

\section{Parasites and isolation of total genomic DNA}

Adult specimens of Fasciola sp. were collected from the liver of a dairy cow (Bos taurus) in Heilongjiang province, China. Adult specimens of $F$. gigantica were collected from the liver of a buffalo (Bubalus bubalis) in Guangxi province, China. The worms were washed extensively in physiological saline, fixed in ethanol and then stored at $-20^{\circ} \mathrm{C}$ until use. Single specimens were identified as Fasciola sp. or F. gigantica based on PCR-based sequencing of the ITS-1 and ITS-2 rDNA regions [11,12].

\section{Long-range PCR-based sequencing of mt DNA}

To obtain some $\mathrm{mt}$ gene sequence data for primer design, regions (400-500 bp) of the cox 1 and nad4 genes were PCR-amplified and sequenced using relatively conserved primers JB3/JB4.5 and ALF/ALR [13,22], respectively. Using BigDye terminator v.3.1 chemistry (Applied Biosystems, Weiterstadt, Germany), the amplicons were sequenced in both directions in a PRISM 3730 sequencer (ABI, USA). After sequencing regions of the cox1 and nad4 genes of both Fasciola sp. and F. gigantica, two internal pairs of conserved primers were designed (Table 1). These pairs were then used to long PCR-amplify the complete mt genome [23] in two overlapping fragments (cox1-nad4; $\sim 9 \mathrm{~kb}$ and, nad4-cox1 = $6 \mathrm{~kb}$ ) from a proportion of total genomic DNA (10-20 ng) from one individual of Fasciola sp. and another of $F$. gigantica. The cycling conditions used were $92^{\circ} \mathrm{C}$ for 2 min (initial denaturation), then $92^{\circ} \mathrm{C}$ for $10 \mathrm{~s}$ (denaturation), $58-63^{\circ} \mathrm{C}$ for $30 \mathrm{~s}$ (annealing), and $60^{\circ} \mathrm{C}$ for $5 \mathrm{~min}$ (extension) for 5 cycles, followed by $92^{\circ} \mathrm{C}$ for $2 \mathrm{~min}, 92^{\circ} \mathrm{C}$ for $10 \mathrm{~s}, 58-63^{\circ} \mathrm{C}$ for $30 \mathrm{~s}$, and $66^{\circ} \mathrm{C}$ for $5 \mathrm{~min}$ for 20 cycles, and a final extension at $66^{\circ} \mathrm{C}$ for $10 \mathrm{~min}$. Each amplicon, which represented a single band in a $0.8 \%(\mathrm{w} / \mathrm{v})$ agarose gel, following electrophoresis and ethidium-bromide staining [23], was column-purified and then sequenced using a primer-walking strategy [24].

\section{Sequence analyses}

Sequences were manually assembled and aligned against each other, and then against the complete $\mathrm{mt}$ genome sequences of 11 other trematodes (see section on Phylogenetic analysis) using the program Clustal X 1.83 [25] and manual adjustment, in order to infer gene boundaries.

Table 1 Sequences of primers used to amplify mt DNA regions from Fasciola spp.

\begin{tabular}{ll}
\hline Primer & Sequence $\left(\mathbf{5}^{\prime}\right.$ to $\left.\mathbf{3}^{\prime}\right)$ \\
\hline F. gigantica & TGTTTACTATTGGTGGGGTTACTGGT \\
FGNR1 & CAAACCCTACAGAACTATCCCTCCAA \\
FGNF1 & GTTATGGGATTCAGTCTTGGAGGGAT \\
FGCR1 & CGTATCCAAAAGAGAAGCAGAAAGCA \\
Fasciola sp. & \\
FZCF1 & GGGTTACTGGTATTATGCTTTCTGCT \\
FZNR1 & CCCTACAGAACTATCCCTCCAAGACT \\
FZNF1 & GGTGGTATTATGGGCAGTATGGGAT \\
FZCR1 & CAGAAAGCATAATACCAGTAACCCCA \\
\hline
\end{tabular}


Open-reading frames (ORFs) were established using the program ORF Finder (http://www.ncbi.nlm.nih.gov/gorf/ gorf.html), employing the trematode mt code, and subsequently compared with those of F. hepatica [26]. Translation initiation and termination codons were identified based on comparisons with those of $F$. hepatica [26]. The secondary structures of 22 tRNA genes were predicted using tRNAscan-SE [27] with manual adjustment [28], and rRNA genes were predicted by comparison with those of $F$. hepatica [26].

\section{Sliding window analysis of nucleotide variation}

To detect variable nucleotide sites, pairwise alignments of the complete genomes, including tRNAs and all intergenic spacers, were performed using Clustal X 1.83. The complete $\mathrm{mt}$ genome sequences of Fasciola sp. and $F$. gigantica were aligned with that published previously for F. hepatica (NC_002546) [26], and sliding window analysis was conducted using DnaSP v.5 [29]. A sliding window of $300 \mathrm{bp}$ (in $10 \mathrm{bp}$ overlapping steps) was used to estimate nucleotide diversity $\mathrm{Pi}(\pi)$ across the alignment. Nucleotide diversity was plotted against mid-point positions of each window, and gene boundaries were identified.

\section{Phylogenetic analysis}

The amino acid sequences conceptually translated from individual genes of the mt genomes of each Fasciola sp. and F. gigantica were concatenated. For comparative purposes, amino acid sequences predicted from published $\mathrm{mt}$ genomes of selected members of the subclass Digenea, including F. hepatica (NC_002546) [26] [Fasciolidae]; Clonorchis sinensis (GeneBank accession no. FJ381664), Opisthorchis felineus (EU921260) [30] and O. viverrini (JF739555) [31] [family Opisthorchiidae]; Paragonimus westermani (NC_002354) [Paragonimidae]; Trichobilharzia regenti (NC_009680) [32], Orientobilharzia turkestanicum (HQ283100) [33], Schistosoma mansoni (NC_002545) [34], S. japonicum (HM120846) [35], S. mekongi (NC_002529) [34], S. spindale (DQ157223) [36] and S. haematobium (DQ157222) [35] [Schistosomatidae], were also included in the analysis. A sequence representing Gyrodactylus derjavinoides (accession no. NC_010976) was included as an outgroup [37]. All amino acid sequences were aligned using the program MUSCLE [38] and subjected to phylogenetic analysis using Bayesian inference (BI), as described previously $[39,40]$. Phylograms were displayed using the program Tree View v.1.65 [41]. In addition, all publicly available sequences of $\mathrm{NADH}$ dehydrogenase subunit 1 gene (nad1) of Fasciola sp.. F. gigantica and F. hepatica were aligned (over a consensus length of $359 \mathrm{bp}$ ) using MUSCLE, the alignment was modified manually, and then subjected to phylogenetic analysis by BI, applying the General Time Reversible (GTR) model. Nodal support values for the final phylogram were determined from the final $75 \%$ of trees obtained using a sample frequency of 100 . The analysis was performed until the potential scale reduction factor approached 1 and the average standard deviation of split frequencies was less than 0.01. An nad1 sequence of Fascioloides magna was used as an outgroup in phylogenetic analysis.

\section{Results}

\section{Identity of the two liver flukes, and features of the} mt genomes

The ITS-1 and ITS-2 sequences (GenBank accession no. KF543341) of the specimen of Fasciola sp. from Heilongjiang province were the same as that of an 'intermediate form' of Fasciola from China (AJ628428, AJ557570 and AJ557571) reported previously $[11,12]$, which is characterized by polymorphic positions at 10 positions in ITS- 1 and ITS-2 (Additional file 1: Figure S1; Table 2). Based on these key polymorphic positions (cf. [11,12]), this specimen of Fasciola sp. from China was inferred to be a hybrid between $F$. gigantica and $F$. hepatica. The ITS-1 and ITS-2 sequences of the $F$. gigantica sample (accession no. KF543340) from Guangxi province were consistent with that of the same species from Niger (AM900371) and did not have any polymorphic positions (Table 2).

The complete mt genome sequences representing Fasciola sp. (GenBank accession no. KF543343) and F. gigantica (accession no. KF543342) were 14,453 bp and 14,478 bp in size, respectively. Each mt genome contains 12 proteincoding genes (cox1-3, nad1-6, nad4L, cytb and atp6), 22 transfer RNA genes and two ribosomal RNA genes ( $r r n S$ and $r r n \mathrm{~L}$ ), but lack an atp 8 gene (Figure 1). The mt genome arrangement of the two flukes is the same as that of F. hepatica [26], but as expected, distinct from Schistosoma spp. [36]. All genes are transcribed in the same direction and have a high $\mathrm{A}+\mathrm{T}$ content (62.7\%). The ATrich regions of both $\mathrm{mt}$ genomes are located between tRNA-Glu and tRNA-Gly, and tRNA-Gly and cox3.

\section{Annotation}

For the two liver flukes, the protein-coding genes were in the following order: $n a d 5>\operatorname{cox} 1>n a d 4>\operatorname{cytb}>\operatorname{nad} 1>$ $n a d 2>\operatorname{cox} 3>\operatorname{cox} 2>\operatorname{atp} 6>\operatorname{nad} 6>\operatorname{nad} 3>\operatorname{nad} 4 \mathrm{~L}$, and the lengths of the all protein-coding genes are the same for Fasciola sp. and F. gigantica (Table 3). The inferred nucleotide and amino acid sequences of each of the $12 \mathrm{mt}$ proteins of two liver flukes were compared. A total of 3,356 amino acids are encoded in the both $\mathrm{mt}$ genomes. All protein-coding genes have ATG, TTG or GTG as their initiation codon (Table 3). All protein-coding genes have TAG as their termination codon, except for cox3 and nad3, which have TAA in Fasciola sp. (Table 3). No abbreviated stop codons, such as TA or T, were detected. Twenty-two tRNA genes were predicted from the $\mathrm{mt}$ genomes of the two liver flukes, and varied from 55 to 
Table 2 Comparison of nucleotides at variable positions in ITS-1 and ITS-2 rDNA sequences of Fasciola from different geographical locations

\begin{tabular}{|c|c|c|c|c|c|c|c|c|c|c|c|c|}
\hline \multirow[t]{2}{*}{ Species } & \multirow[t]{2}{*}{ Locations } & \multicolumn{10}{|c|}{ Variable positions in ITS- 1 and ITS- 2 sequences ${ }^{*}$} & \multirow[t]{2}{*}{ Accession nos. } \\
\hline & & 18 & 108 & 202 & 280 & 300 & 791 & 815 & 854 & 860 & 911 & \\
\hline \multirow[t]{5}{*}{ F. hepatica } & China & $C$ & $A$ & C & $\mathrm{T}$ & C & $\mathrm{T}$ & $\mathrm{T}$ & C & C & $\mathrm{T}$ & JF708026 \\
\hline & France & C & A & C & $\mathrm{T}$ & C & $\mathrm{T}$ & $\mathrm{T}$ & C & C & $\mathrm{T}$ & JF708034 \\
\hline & Iran & C & A & C & $\mathrm{T}$ & C & $\mathrm{T}$ & $\mathrm{T}$ & C & C & $\mathrm{T}$ & JF432072 \\
\hline & Niger & C & A & C & $\mathrm{T}$ & C & $\mathrm{T}$ & $\mathrm{T}$ & C & C & $\mathrm{T}$ & AM850107 \\
\hline & Spain & C & A & C & $\mathrm{T}$ & C & $\mathrm{T}$ & $\mathrm{T}$ & C & C & $\mathrm{T}$ & JF708036 \\
\hline \multirow[t]{4}{*}{ F. gigantica } & Burkina Faso & T & $\mathrm{T}$ & $\mathrm{T}$ & A & $\mathrm{T}$ & C & C & $\mathrm{T}$ & $\mathrm{T}$ & - & AJ853848 \\
\hline & China & T & $\mathrm{T}$ & $\mathrm{T}$ & A & $\mathrm{T}$ & C & C & $\mathrm{T}$ & $\mathrm{T}$ & - & JF496709 \\
\hline & Niger & T & $\mathrm{T}$ & $\mathrm{T}$ & A & $\mathrm{T}$ & C & C & $\mathrm{T}$ & $\mathrm{T}$ & - & AM900371 \\
\hline & Present study & T & $\mathrm{T}$ & $\mathrm{T}$ & A & $\mathrm{T}$ & C & C & $\mathrm{T}$ & $\mathrm{T}$ & - & KF543340 \\
\hline \multirow[t]{3}{*}{ Fasciola sp. } & China & $\mathrm{C} / \mathrm{T}$ & $\mathrm{A} / \mathrm{T}$ & $\mathrm{C} / \mathrm{T}$ & $\mathrm{T} / \mathrm{A}$ & $\mathrm{C} / \mathrm{T}$ & $\mathrm{T} / \mathrm{C}$ & $\mathrm{T} / \mathrm{C}$ & $\mathrm{C} / \mathrm{T}$ & $\mathrm{C} / \mathrm{T}$ & $\mathrm{T} /-$ & AJ628428, AJ557570, AJ557571 \\
\hline & China, Japan & C & A & C & $\mathrm{T}$ & C & $\mathrm{T}$ & $\mathrm{T}$ & C & C & $\mathrm{T}$ & AB385611, AB010978 \\
\hline & Present study & $\mathrm{C} / \mathrm{T}$ & $\mathrm{A} / \mathrm{T}$ & $\mathrm{C} / \mathrm{T}$ & $\mathrm{T} / \mathrm{A}$ & $\mathrm{C} / \mathrm{T}$ & $\mathrm{T} / \mathrm{C}$ & $\mathrm{T} / \mathrm{C}$ & $\mathrm{C} / \mathrm{T}$ & $\mathrm{C} / \mathrm{T}$ & $\mathrm{T}$ & KF543341 \\
\hline
\end{tabular}

*Sequence positions were determined by comparison with that of a previous study [11]. Sequences include ITS-1 (polymorphic positions 18, 108, 202, 280, 300), 5.8S rDNA and ITS-2 (polymorphic positions 791, 815, 854, 860, 911).

$69 \mathrm{bp}$ in size. Of all tRNA genes, 20 can be folded into the conventional four-arm cloverleaf structures. The tRNA-tRNA-Ser ${ }^{(\mathrm{UCN})}$ and tRNA-Ser ${ }^{(\mathrm{AGN})}$ show unorthodox structures; their D-arms are unpaired and replaced by the loops of 8-11 bp.

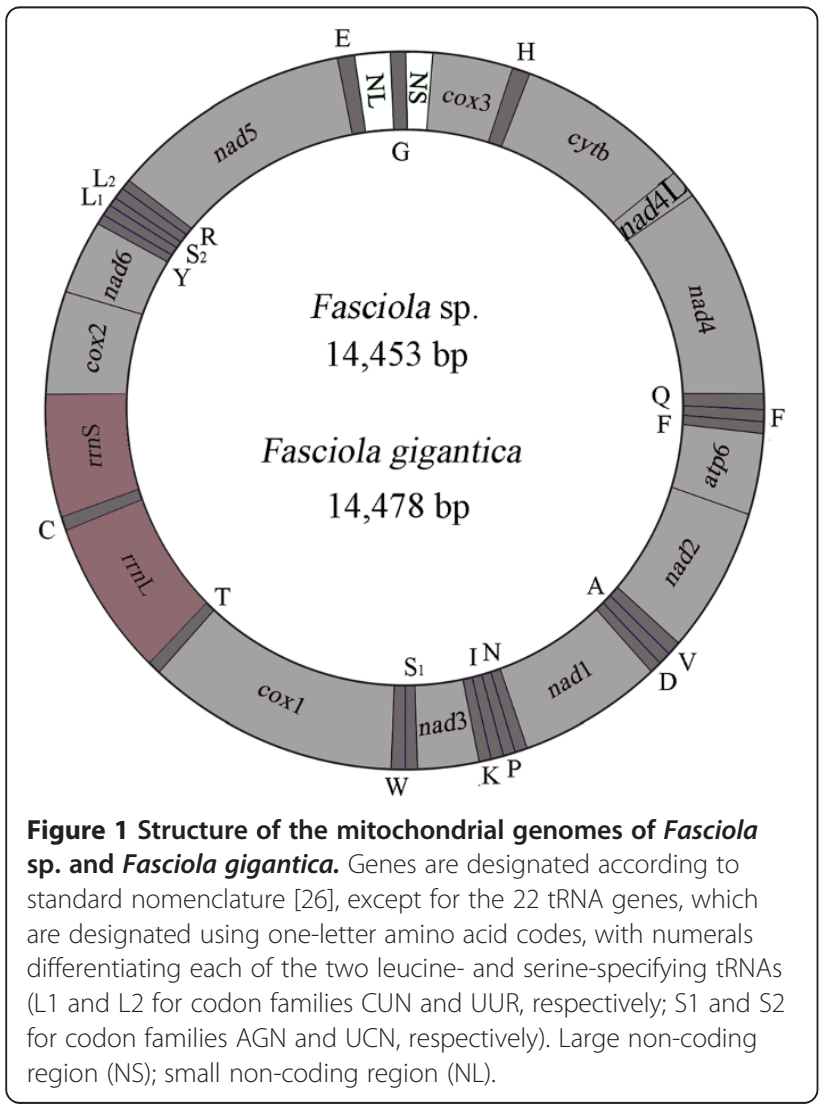

The two ribosomal RNA genes ( $r r n \mathrm{~L}$ and $r r n S$ ) of Fasciola sp. and F. gigantica were inferred based on comparisons with sequences from those of $F$. hepatica. The rrnL of Fasciola sp. and F. gigantica is located between tRNA-Thr and tRNA-Cys, and $r r n S$ is located between tRNA-Cys and cox2. The length of $r r n \mathrm{~L}$ is 987 bp for both Fasciola sp. and F. gigantica. The size of the $r r n S$ genes is $769 \mathrm{bp}$ and $771 \mathrm{bp}$ for Fasciola sp. and F. gigantica, respectively. The A + T contents of $r r n \mathrm{~L}$ and $r r n S$ are $\sim 62 \%$ and $\sim 61 \%$ for Fasciola sp. and $F$. gigantica, respectively.

Two AT-rich non-coding regions (NCR) in the mt genomes Fasciola sp. and F. gigantica were inferred. In both mt genomes, the long NCR (841 bp) is located between the tRNA-Gly and cox3 (Figure 1), has an A + T content of $\sim 53 \%$ and contains eight perfect, 86 bp tandem repeats (TR1 to TR8). The short NCR is $174-176$ bp in length, is located between tRNA-Glu and tRNA-Gly (Figure 1) and has an $\mathrm{A}+\mathrm{T}$ content of $\sim 72 \%$.

\section{Comparative mt genomic analyses of Fasciola sp. and}

\section{F. gigantica with $F$. hepatica}

The complete mt genome sequences representing Fasciola sp. and F. gigantica are 9 bp shorter and 16 bp longer than $F$. hepatica (14,462 bp in length) [26], respectively. A comparison of the nucleotide sequences of each $\mathrm{mt}$ gene, and the amino acid sequences, conceptually translated from all $\mathrm{mt}$ protein-encoding genes of the three flukes, is given in Table 4. Across the entire mt genome, the sequence difference was $2.6 \%$ (380 nucleotide substitutions) between Fasciola sp. and F. gigantica, 11.8\% (1712 nucleotide substitutions) between Fasciola sp. and $F$. hepatica, and $11.8 \%$ (1714 nucleotide substitutions) 
Table 3 The organization of the mt genomes of Fasciola sp., Fasciola gigantica and F. hepatica

\begin{tabular}{|c|c|c|c|c|c|c|}
\hline \multirow[t]{2}{*}{ Genes } & \multicolumn{3}{|c|}{ Positions and nt sequence lengths (bp) } & \multicolumn{3}{|c|}{ Ini/Ter codons } \\
\hline & Fasciola sp. & Fasciola gigantica & Fasciola hepatica & Fasciola sp. & Fasciola gigantica & Fasciola hepatica \\
\hline $\cos 3$ & $1-642$ & $1-642$ & $1-642$ & ATG/TAA & ATG/TAG & ATG/TAG \\
\hline tRNA-His & $650-713(64)$ & 650-713 (64) & $650-713(64)$ & & & \\
\hline cytb & 715-1827 & $715-1827(62)$ & 715-1827 & ATG/TAG & ATG/TAG & ATG/TAG \\
\hline nad4L & 1836-2108 & $1836-2108$ & $1836-2108$ & GTG/TAG & GTG/TAG & GTG/TAG \\
\hline nad4 & 2069-3337 & 2069-3337 & 2069-3340 & GTG/TAG & GTG/TAG & GTG/TAA \\
\hline tRNA-GIn & $3339-3404(66)$ & $3339-3404(66)$ & $3342-3404(63)$ & & & \\
\hline tRNA-Phe & $3420-3484(65)$ & $3417-3481(65)$ & $3417-3482(66)$ & & & \\
\hline tRNA-Met & $3491-3556(66)$ & $3488-3553(66)$ & $3494-3561(68)$ & & & \\
\hline atp6 & $3557-4075$ & $3554-4072$ & $3562-4080$ & ATG/TAG & ATG/TAG & ATG/TAG \\
\hline nad2 & $4088-4954$ & $4085-4951$ & 4093-4959 & ATG/TAG & ATG/TAG & ATG/TAG \\
\hline tRNA-Val & 4959-5021 (63) & $4957-5020(64)$ & $4965-5027(63)$ & & & \\
\hline tRNA-Ala & 5035-5099 (65) & $5035-5099(65)$ & $5042-5104(63)$ & & & \\
\hline tRNA-Asp & 5103-5167 (65) & $5103-5167(65)$ & $5107-5172(66)$ & & & \\
\hline nad1 & $5171-6073$ & $5171-6073$ & $5176-6078$ & GTG/TAG & GTG/TAG & GTG/TAG \\
\hline tRNA-Asn & 6079-6146 (68) & $6084-6153(70)$ & 6089-6158 (70) & & & \\
\hline tRNA-Pro & $6152-6220(69)$ & $6163-6230(68)$ & $6168-6234(67)$ & & & \\
\hline tRNA-Ile & $6221-6282(62)$ & $6231-6292(62)$ & $6235-6296(62)$ & & & \\
\hline tRNA-Lys & $6287-6352(66)$ & $6297-6363(67)$ & $6301-6367(67)$ & & & \\
\hline nad3 & 6353-6709 & $6364-6720$ & $6368-6724$ & ATG/TAG & ATG/TAG & ATG/TAG \\
\hline tRNA-Ser UCN & $6714-6768(55)$ & $6725-6780(56)$ & $6731-6788(58)$ & & & \\
\hline tRNA-Trp & $6771-6833(63)$ & $6790-6852(63)$ & $6796-6858(63)$ & & & \\
\hline $\operatorname{cox} 1$ & 6837-8378 & 6865-8397 & $6871-8403$ & GTG/TAG & GTG/TAG & ATG/TAG \\
\hline tRNA-Thr & $8391-8458$ (68) & 8419-8486 (68) & $8420-8488$ (69) & & & \\
\hline$r r n \mathrm{~L}$ & 8460-9445 & $8488-9473$ & 8489-9475 & & & \\
\hline tRNA-Cys & $9446-9510(65)$ & $9474-9538(65)$ & $9476-9538(63)$ & & & \\
\hline$r r n S$ & 9511-10279 & 9539-10309 & 9539-10304 & & & \\
\hline $\cos 2$ & 10280-10882 & 10310-10912 & 10305-10907 & ATG/TAA & ATG/TAG & ATG/TAG \\
\hline nad6 & 10929-11381 & 10959-11411 & 10950-11402 & ATG/TAG & ATG/TAG & ATG/TAG \\
\hline tRNA-Tyr & $11389-11445$ (57) & $11419-11475$ (57) & $11411-11467(67)$ & & & \\
\hline tRNA-Leu ${ }^{\text {CUN }}$ & $11456-11520$ (65) & $11486-11550(65)$ & 11478-11543 (66) & & & \\
\hline tRNA-Ser ${ }^{A G N}$ & $11521-11579(59)$ & $11551-11607$ (57) & $11542-11603(62)$ & & & \\
\hline tRNA-Leu UUR & $11588-11651$ (64) & $11616-11678$ (63) & $11609-11673(64)$ & & & \\
\hline tRNA-Arg & $11653-11718$ (66) & $11680-11745$ (66) & $11673-11738(66)$ & & & \\
\hline nad5 & 11720-13282 & 11747-13309 & 11737-13305 & TTG/TAG & TTG/TAG & GTG/TAG \\
\hline tRNA-Glu & $13305-13372(68)$ & $13332-13399(68)$ & 13327-13395 (69) & & & \\
\hline Short non-coding region & 13373-13548 (176) & 13400-13573 (174) & 13396-13582 (187) & & & \\
\hline tRNA-Gly & $13549-13612(64)$ & 13574-13637 (64) & $13583-13645(63)$ & & & \\
\hline Long non-coding region & $13613-14453(841)$ & $13638-14478$ (841) & $13646-14462$ (817) & & & \\
\hline
\end{tabular}

between $F$. gigantica and $F$. hepatica. The difference across both nucleotide and amino acid sequences of the 12 protein-coding was $11.6 \%$ (1167 nucleotide substitutions) and 9.54\% (320 amino acid substitutions) between the Fasciola sp. and F. hepatica; 11.6\% (1167 nucleotide substitutions) and 9.83\% (330 amino acid substitutions) between the F. gigantica and F. hepatica; and $2.8 \%$ (281 nucleotide substitutions) and 2.1\% (71 amino acid substitutions) between the Fasciola sp. and F. gigantica, respectively. 
Table 4 Nucleotide (nt) and/or predicted amino acid (aa) sequence differences in each mt gene among Fasciola sp. (F), Fasciola gigantica $(\mathrm{Fg})$ and $\boldsymbol{F}$. hepatica $(\mathrm{Fh})$ upon pairwise comparison

\begin{tabular}{|c|c|c|c|c|c|c|c|c|c|c|c|c|}
\hline \multirow[t]{2}{*}{ Gene/region } & \multicolumn{3}{|c|}{ Nt sequence length } & \multicolumn{3}{|c|}{ Nt difference (\%) } & \multicolumn{3}{|c|}{ Number of aa } & \multicolumn{3}{|c|}{ aa difference (\%) } \\
\hline & $F$ & $\mathrm{Fg}$ & $\mathrm{Fh}$ & $\mathrm{F} / \mathrm{Fg}$ & F/Fh & $\mathrm{Fg} / \mathrm{Fh}$ & $F$ & $\mathrm{Fg}$ & $\mathrm{Fh}$ & $\mathrm{F} / \mathrm{Fg}$ & F/Fh & $\mathrm{Fg} / \mathrm{Fh}$ \\
\hline atp6 & 519 & 519 & 519 & 2.89 & 15.22 & 13.87 & 172 & 172 & 172 & 1.74 & 15.12 & 13.95 \\
\hline nad1 & 903 & 903 & 903 & 3.10 & 8.86 & 8.42 & 300 & 300 & 300 & 2.67 & 7.67 & 8.0 \\
\hline nad2 & 867 & 867 & 867 & 3.69 & 11.42 & 11.65 & 288 & 288 & 288 & 1.74 & 11.81 & 11.81 \\
\hline nad3 & 357 & 357 & 357 & 5.60 & 10.64 & 10.64 & 118 & 118 & 118 & 0.85 & 7.63 & 7.63 \\
\hline nad4 & 1269 & 1269 & 1272 & 3.86 & 13.99 & 13.68 & 422 & 422 & 423 & 3.08 & 11.58 & 11.11 \\
\hline nad4L & 273 & 273 & 273 & 1.83 & 8.79 & 8.42 & 90 & 90 & 90 & 2.22 & 5.56 & 5.56 \\
\hline nad5 & 1563 & 1563 & 1569 & 1.86 & 13.58 & 14.02 & 520 & 520 & 522 & 1.35 & 12.45 & 12.45 \\
\hline nad6 & 453 & 453 & 453 & 3.97 & 13.91 & 16.34 & 150 & 150 & 150 & 7.33 & 8.00 & 14.67 \\
\hline $\operatorname{cox} 1$ & 1542 & 1542 & 1533 & 2.02 & 9.39 & 9.13 & 513 & 513 & 510 & 1.37 & 6.08 & 5.49 \\
\hline $\cos 2$ & 603 & 603 & 603 & 2.16 & 11.11 & 11.61 & 200 & 200 & 200 & 0.50 & 7.00 & 7.50 \\
\hline $\operatorname{cox} 3$ & 642 & 642 & 642 & 2.80 & 13.86 & 13.40 & 213 & 213 & 213 & 2.82 & 14.55 & 14.55 \\
\hline cytb & 1113 & 1113 & 1113 & 2.07 & 8.36 & 8.36 & 370 & 370 & 370 & 1.89 & 6.22 & 7.03 \\
\hline rrns & 769 & 771 & 766 & 1.30 & 11.31 & 11.41 & & - & - & & - & \\
\hline$r r n \mathrm{~L}$ & 986 & 986 & 987 & 1.01 & 9.93 & 10.13 & & - & - & & - & \\
\hline 22 tRNAs & 1413 & 1414 & 1420 & 2.26 & 10.28 & 10.63 & & & & & & \\
\hline
\end{tabular}

Nucleotide variability in the $\mathrm{mt}$ genome among Fasciola sp., F. gigantica and F. hepatica

Sliding window analysis across the mt genomes of Fasciola sp., F. gigantica and F. hepatica provided an estimation of nucleotide diversity $\mathrm{Pi}(\pi)$ for individual $\mathrm{mt}$ genes (Figure 2). By computing the number of variable positions per unit length of gene, the sliding window indicated that the highest and lowest levels of sequence variability were within the genes nad6 and cytb, respectively. Conserved regions were identified within $n a d 1$ and cox 1 genes. In this study, the cytb and $n a d 1$ genes are the most conserved protein-coding genes, and nad6, nad5 and nad4 are the least conserved.

\section{Phylogenetic analysis}

Phylogenetic analysis of the concatenated amino acid sequence data for all $12 \mathrm{mt}$ proteins (Figure 3) showed that the Fasciolidae clustered to the exclusion of representatives of the families Paragonimidae ( $P$. westermani) and Opisthorchiidae (O. viverrini, O. felineus and C. sinensis); the Schistosomatidae clustered separately with strong nodal support (posterior probability $(\mathrm{pp})=1.0$ ). Within the Fasciolidae, Fasciola sp. and F. gigantica clustered together with strong support ( $\mathrm{pp}=1.0)$, to the exclusion of $F$. hepatica, with the former two taxa being more closely related than either was to $F$. hepatica. In addition, phylogenetic analysis using the nad 1 data supports clustering of

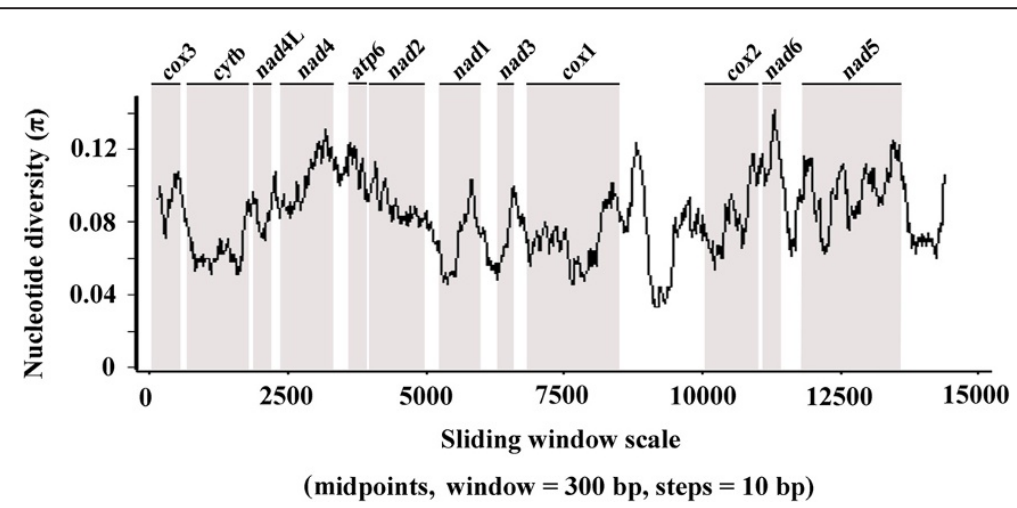

Figure 2 Sliding window analysis of complete $\mathrm{mt}$ genome sequences of Fasciola sp., Fasciola gigantica and $F$. hepatica. The black line indicates nucleotide diversity in a window of 300 bp (10 bp-steps). Gene regions (grey) and boundaries are indicated. 


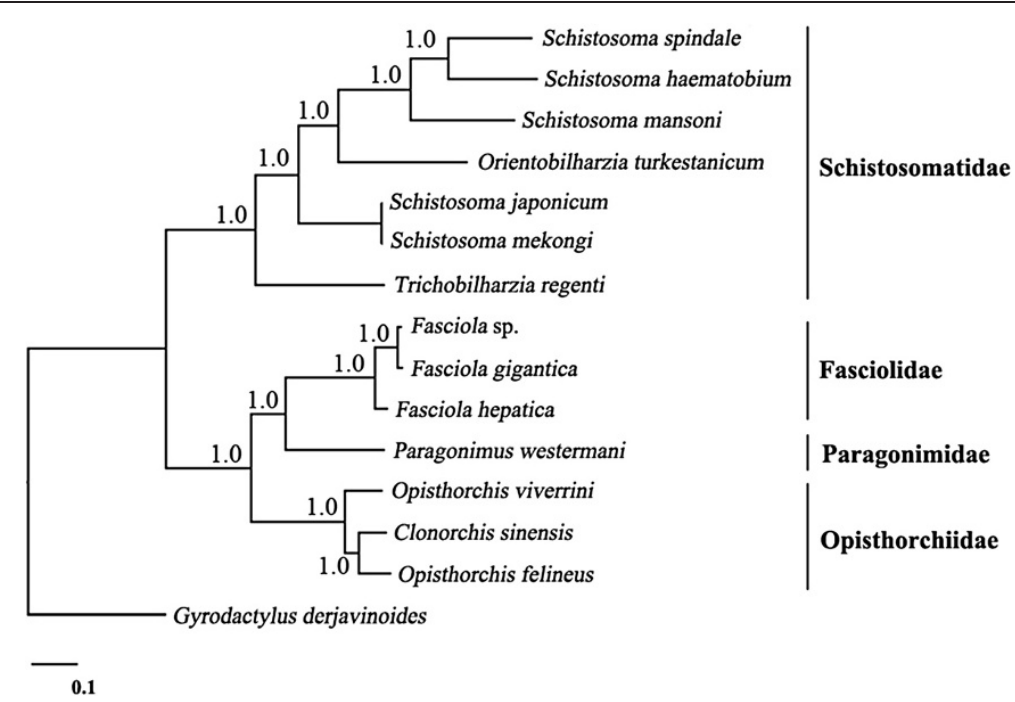

Figure 3 Genetic relationships of Fasciola sp. with Fasciola gigantica and F. hepatica, and other trematodes. Phylogenetic analysis of the concatenated amino acid sequence data representing 12 protein-coding genes was conducted using Bayesian inference (BI), using Gyrodacty/us derjavinoides (NC_010976) as an outgroup.

the Fasciola sp. with aspermic F. gigantica x F. hepatica hybrids characterised previously [42] (Additional file 2: Figure S2).

\section{Discussion}

The present comparative, genetic investigation of representative specimens of Fasciola sp. (i.e. the 'intermediate form'), F. gigantica and F. hepatica using whole $\mathrm{mt}$ genomic and protein sequence data sets showed that Fasciola sp. and F. gigantica were more closely related than either was to F. hepatica. This finding was also supported by an analysis of nad1 sequence data (cf. Additional file 2: Figure S2). Although this evidence might suggest that Fasciola sp. is a population variant of $F$. gigantica, previous studies [19-21] have proposed that Fasciola sp. is a hybrid of F. gigantica and F. hepatica. The combined use of mtDNA (if indeed maternally inherited in fasciolids) and nuclear DNA markers [43] should assist in exploring the "hybridization/speciation" hypotheses [44]. Clearly, there is consistent evidence from various studies $[11,12,14]$ of mixed ITS-1 and ITS-2 sequence types, representing both $F$. gigantica and $F$. hepatica among the multiple rDNA copies, within individual specimens of Fasciola sp. (i.e., the 'intermediate form'). Although the number or proportion(s) of different sequence types within individual adults of Fasciola sp. has not yet been estimated using a mutation scanning- or cloning-based sequencing [45], the polymorphic positions in the sequences determined by direct sequencing $[11,14]$ indicate a clear pattern of introgression between the $F$. gigantica and $F$. hepatica. Although $\mathrm{mt}$ genomic (11.8\%) and inferred protein (9.83\%) sequence differences between these two species is substantial, the explanation that
Fasciola sp. represents a hybrid between these two recognized species seems plausible, given that the karyotypes of both diploid F. hepatica and F. gigantica are the same $(2 \mathrm{n}=20) \quad[46,47]$ and that the magnitude of sequence variation (1.7\%) in ITS-2 (a species marker) between $F$. gigantica and $F$. hepatica is comparable with the highest level (1.3-1.6\%) in this rDNA region between some schistosome species for which hybrids (i.e. S. haematobium $\times$ S. bovis; S. haematobium $\times$ S. guineenis; S. haematobium $\times$ S. intercalatum) have been reported [48-50]. While hybridization seems possible, another explanation might be ITS rDNA "lineage sorting and retention of ancestral polymorphism" [51,52], but this is perhaps less likely, given a clear pattern of mixing of ITS sequences seen in Fasciola sp. (cf. Additional file 1: Figure S1).

In addition, polyploidy or diploidy in aspermic Fasciola [20] needs to be considered, and warrants future investigation. Perhaps the aspermic Fasciola specimens described in the literature [53] were infertile hybrids of $F$. gigantica and $F$. hepatica (in situations where both species occur in sympatry). Questions that might be addressed directly in relation to Fasciola sp. are: Are eggs from Fasciola sp. fertilized and viable? If miracidia develop and emerge from these eggs, are they infective to snails? If they do infect snails, do the ensuing adult worms (in the definitive host) contain sperm and are these worms fertile, and what is their ploidy? These questions should be addressed, and could be complemented by detailed light and transmission electron microscopic investigations of a relatively large number of adult specimens of Fasciola sp., F. gigantica and $F$. hepatica (preferably from different countries), which have been unequivocally and individually identified based on their ITS- 1 and ITS- 2 sequences. Such a study 
should pay particular attention to the morphology of the reproductive organs, sperm and oocytes, and the karyotypes of worms, and establish whether or not Fasciola sp. from China is polyploid and/or aspermic [20].

Moreover, although challenging, laborious and timeconsuming, it would be highly informative to conduct hybridization studies in vivo, whereby individual miracidia from eggs from adults of each Fasciola sp., F. gigantica and $F$. hepatica would be used to infect (separately) their lymnaeid snail hosts, to raise clonal populations of cercariae and metacercariae of these three taxa, so that mixed infections (in different combinations and with monospecific controls) could be established in, for example, sheep or goats, to attempt to cross-hybridize the three taxa in a pairwise manner. Using such an experimental design, eggs and adult worms could then be examined in detail at both the electron microscopic, karyotypic and molecular levels. Importantly, in these experiments, ITS-1 and/or ITS-2 could be used to establish the genotypes of subsamples of individuals, and mt markers derived from $\mathrm{mt}$ genomes determined here and of $F$. hepatica could be used to determine haplotypes and mtDNA inheritance if the cross-hybridization studies were successful. Therefore, the present markers could be employed, in combination, to establish the biological relationship of the three taxa through in vivo experiments, but also in the field in sympatric and allopatric populations, if they occur. Combined with the use of markers in nuclear and mt genomes, advanced genomic sequencing, optical mapping and microimaging techniques might assist studies of Fasciola sp. in China and other countries.

\section{Conclusion}

The findings of this study provide robust genetic evidence that Fasciola sp. is more closely related to F. gigantica than to $F$. hepatica. The mtDNA datasets reported in the present study should provide useful novel markers for further studies of the taxonomy and systematics of Fasciola from different hosts and geographical regions.

\section{Additional files}

Additional file 1: Figure S1. Polymorphic positions in the interna transcribed spacer regions (ITS-1 and ITS-2) of nuclear ribosomal DNA of Fasciola sp.

Additional file 2: Figure S2. Phylogenetic tree of Fasciola spp. inferred from the mitochondrial nad1 sequence data by Bayesian inference (BI). Fascioloides magna was used as an outgroup. Nodal support values were determined from the final $75 \%$ of trees using a sampling frequency of 100 .

\section{Competing interests}

The authors declare that they have no competing interests.

\section{Authors' contributions}

GHL, NY, HQS and LA performed the experiments, analyzed the data and drafted parts of the manuscript. XQZ and RBG revised and edited the manuscript and funded the study. All authors read and approved the final manuscript.

\section{Acknowledgements}

This work was supported in part by the International Science \& Technology Cooperation Program of China (Grant No. 2013DFA31840), the "Special Fund for Agro-scientific Research in the Public Interest" (Grant No. 201303037), and the Science Fund for Creative Research Groups of Gansu Province (Grant No. 1210RJIA006). RBG's research is supported by the Australian Research Council (ARC), National Health and Medical Research Council (NHMRC) and Melbourne Water Corporation (MWC); the Alexander von Humboldt Foundation is also gratefully acknowledged. This study was also supported by a Victorian Life Sciences Computation Initiative (VLSCI) grant number VR0007 on its Peak Computing Facility at the University of Melbourne, an initiative of the Victorian Government. NDY is an NHMRC Early Career Research Fellow.

\section{Author details}

'State Key Laboratory of Veterinary Etiological Biology, Key Laboratory of Veterinary Parasitology of Gansu Province, Lanzhou Veterinary Research Institute, Chinese Academy of Agricultural Sciences, Lanzhou, Gansu Province, PR China. ${ }^{2}$ Faculty of Veterinary Science, The University of Melbourne, Parkville, Victoria, Australia. ${ }^{3}$ College of Veterinary Medicine, Hunan Agricultural University, Changsha, Hunan Province, PR China. ${ }^{4}$ National Institute of Parasitic Diseases, Chinese Center for Disease Control and Prevention, Shanghai, PR China.

Received: 11 March 2014 Accepted: 15 March 2014

Published: 31 March 2014

\section{References}

1. Fürst T, Keiser J, Utzinger J: Global burden of human food-borne trematodiasis: a systematic review and meta-analysis. Lancet Infect Dis 2012, 12:210-221.

2. Sripa B: Global burden of food-borne trematodiasis. Lancet Infect Dis 2012, 12:171-172.

3. Mas-Coma S, Bargues MD, Valero MA: Fascioliasis and other plant-borne trematode zoonoses. Int J Parasitol 2005, 35:1255-1278.

4. Zhou P, Chen N, Zhang RL, Lin RQ, Zhu XQ: Food-borne parasitic zoonoses in China: perspective for control. Trends Parasitol 2008, 24:190-196.

5. Ai L, Chen MX, Alasaad S, Elsheikha HM, Li J, Li HL, Lin RQ, Zou FC, Zhu XQ, Chen JX: Genetic characterization, species differentiation and detection of Fasciola spp. by molecular approaches. Parasit Vectors 2011, 4:101.

6. Keiser J, Engels D, Büscher G, Utzinger J: Triclabendazole for the treatment of fascioliasis and paragonimiasis. Expert Opin Investig Drugs 2005, 14:1513-1526.

7. Brennan GP, Fairweather I, Trudgett A, Hoey E, McCoy, McConville M, Meaney M, Robinson M, McFerran N, Ryan L, Lanusse C, Mottier L, Alvarez L, Solana H, Virkel G, Brophy PM: Understanding triclabendazole resistance. Exp Mol Pathol 2007, 82:104-109.

8. Heneberg P: Phylogenetic data suggest the reclassification of Fasciola jacksoni (Digenea: Fasciolidae) as Fascioloides jacksoni comb. nov. Parasitol Res 2013, 112:1679-1689.

9. Reddy PV, Subramanyam S: Chromosome studies in the liver fluke, Fasciola gigantica Cobbold, 1856, from Andra Pradesh. Curr Sci 1973, 42:288-291.

10. Periago MV, Valero MA, El Sayed M, Ashrafi K, El Wakeel A, Mohamed MY, Desquesnes M, Curtale F, Mas-Coma S: First phenotypic description of Fasciola hepatica/Fasciola gigantica intermediate forms from the human endemic area of the Nile Delta, Egypt. Infect Genet Evol 2008, 8:51-58.

11. Ali H, Ai L, Song HQ, Ali S, Lin RQ, Seyni B, Issa G, Zhu XQ: Genetic characterisation of Fasciola samples from different host species and geographical localities revealed the existence of $F$. hepatica and $F$. gigantica in Niger. Parasitol Res 2008, 102:1021-1024.

12. Huang WY, He B, Wang CR, Zhu XQ: Characterisation of Fasciola species from Mainland China by ITS-2 ribosomal DNA sequence. Vet Parasitol 2004, 120:75-83. 
13. Ai L, Weng YB, Elsheikha HM, Zhao GH, Alasaad S, Chen JX, Li J, Li HL, Wang $C R$, Chen MX, Lin RQ, Zhu XQ: Genetic diversity and relatedness of Fasciola spp. isolates from different hosts and geographic regions revealed by analysis of mitochondrial DNA sequences. Vet Parasitol 2011, 181:329-334.

14. Choe SE, Kang TG, Kweon CH, Kang SW: Genetic analysis of Fasciola isolates from cattle in Korea based on second internal transcribed spacer (ITS-2) sequence of nuclear ribosomal DNA. Parasitol Res 2011, 109:833-839.

15. Amor N, Halajian A, Farjallah S, Merella P, Said K, Ben Slimane B: Molecular characterization of Fasciola spp. from the endemic area of northern Iran based on nuclear ribosomal DNA sequences. Exp Parasitol 2011, 128:196-204.

16. Itagaki T, Kikawa M, Sakaguchi K, Shimo J, Terasaki K, Shibahara T, Fukuda K: Genetic characterization of parthenogenic Fasciola sp. in Japan on the basis of the sequences of ribosomal and mitochondrial DNA. Parasitology 2005, 131:679-685.

17. Alasaad S, Huang CQ, Li QY, Granados JE, García-Romero C, Pérez JM, Zhu XQ: Characterization of Fasciola samples from different host species and geographical localities in Spain by sequences of internal transcribed spacers of rDNA. Parasitol Res 2007, 101:1245-1250.

18. Farjallah S, Sanna D, Amor N, Ben Mehel B, Piras MC, Merella P, Casu M, Curini-Galletti M, Said K, Garippa G: Genetic characterization of Fasciola hepatica from Tunisia and Algeria based on mitochondrial and nuclear DNA sequences. Parasitol Res 2009, 105:1617-1621.

19. Agatsuma T, Arakawa Y, Iwagami M, Honzako Y, Cahyaningsih U, Kang SY, Hong SJ: Molecular evidence of natural hybridization between Fasciola hepatica and F. gigantica. Parasitol Int 2000, 49:231-238.

20. Itagaki T, Arakawa Y, Iwagami M, Honzako Y, Cahyaningsih U, Kang SY, Hong SJ: Occurrence of spermic diploid and aspermic triploid forms of Fasciola in Vietnam and their molecular characterization based on nuclear and mitochondrial DNA. Parasitol Int 2009, 58:81-85.

21. Le TH, De NV, Agatsuma T, Thi Nguyen TG, Nguyen QD, McManus DP, Blair D: Human fascioliasis and the presence of hybrid/introgressed forms of Fasciola hepatica and Fasciola gigantica in Vietnam. Int J Parasitol 2008, 38:725-730.

22. Bowles J, Blair D, McManus DP: Genetic variants within the genus Echinococcus identified by mitochondrial DNA sequencing. Mol Biochem Parasitol 1992, 54:165-173.

23. Liu GH, Gasser RB, Su A, Nejsum P, Peng L, Lin RQ, Li MW, Xu MJ, Zhu XQ. Clear genetic distinctiveness between human- and pig-derived Trichuris based on analyses of mitochondrial datasets. PLOS Negl Trop Dis 2012, 6:e1539.

24. Hu M, Jex AR, Campbell BE, Gasser RB: Long PCR amplification of the entire mitochondrial genome from individual helminths for direct sequencing. Nat Protoc 2007, 2:2339-2344.

25. Thompson JD, Gibson TJ, Plewniak F, Jeanmougin F, Higgins DG: The Clustal X windows interface: flexible strategies for multiple sequence alignment aided by quality analysis tools. Nucleic Acids Res 1997, 24:4876-4882

26. Le TH, Blair D, McManus DP: Complete DNA sequence and gene organization of the mitochondrial genome of the liver fluke, Fasciola hepatica L. (Platyhelminthes; Trematoda). Parasitology 2001, 123:609-621.

27. Lowe TM, Eddy SR: tRNAscan-SE: A program for improved detection of transfer RNA genes in genomic sequence. Nucleic Acids Res 1997, 25:955-964

28. Hu M, Chilton NB, Gasser RB: The mitochondrial genomes of the human hookworms, Ancylostoma duodenale and Necator americanus (Nematoda: Secernentea). Int J Parasitol 2002, 32:145-158.

29. Librado P, Rozas J: DnaSP v5: a software for comprehensive analysis of DNA polymorphism data. Bioinformatics 2009, 25:1451-1452.

30. Shekhovtsov SV, Katokhin AV, Kolchanov NA, Mordvinov VA: The complete mitochondrial genomes of the liver flukes Opisthorchis felineus and Clonorchis sinensis (Trematoda). Parasitol Int 2010, 59:100-103.

31. Cai XQ, Liu GH, Song HQ, Wu CY, Zou FC, Yan HK, Yuan ZG, Lin RQ, Zhu XQ: Sequences and gene organization of the mitochondrial genomes of the liver flukes Opisthorchis viverrini and Clonorchis sinensis (Trematoda). Parasitol Res 2012, 110:235-243.

32. Webster BL, Rudolfová J, Horák P, Littlewood DT: The complete mitochondrial genome of the bird schistosome Trichobilharzia regenti (Platyhelminthes: Digenea), causative agent of cercarial dermatitis. J Parasitol 2007, 93:553-561.
33. Wang Y, Wang CR, Zhao GH, Gao JF, Li MW, Zhu XQ: The complete mitochondrial genome of Orientobilharzia turkestanicum supports its affinity with African Schistosoma spp. Infect Genet Evol 2011, 11:1964-1970.

34. Le TH, Blair D, Agatsuma T, Humair PF, Campbell NJ, Iwagami M, Littlewood DT, Peacock B, Johnston DA, Bartley J, Rollinson D, Herniou EA, Zarlenga DS, McManus DP: Phylogenies inferred from mitochondrial gene orders-a cautionary tale from the parasitic flatworms. Mol Biol Evol 2000, 17:1123-1125.

35. Zhao GH, Li J, Song HQ, Li XY, Chen F, Lin RQ, Yuan ZG, Weng YB, Hu M, Zou FC, Zhu XQ: A specific PCR assay for the identification and differentiation of Schistosoma japonicum geographical isolates in mainland China based on analysis of mitochondrial genome sequences. Infect Genet Evol 2012, 12:1027-1036.

36. Littlewood DT, Lockyer AE, Webster BL, Johnston DA, Le TH: The complete mitochondrial genomes of Schistosoma haematobium and Schistosoma spindale and the evolutionary history of mitochondrial genome changes among parasitic flatworms. Mol Phylogenet Evol 2006, 39:452-467.

37. Huyse T, Buchmann K, Littlewood DT: The mitochondrial genome of Gyrodactylus derjavinoides (Platyhelminthes: Monogenea)-a mitogenomic approach for Gyrodactylus species and strain identification. Gene 2008, 417:27-34.

38. Edgar RC: MUSCLE: multiple sequence alignment with high accuracy and high throughput. Nucleic Acids Res 2004, 32:1792-1797.

39. Liu GH, Wang SY, Huang WY, Zhao GH, Wei SJ, Song HQ, Xu MJ, Lin RQ, Zhou DH, Zhu XQ: The complete mitochondrial genome of Galba pervia (Gastropoda: Mollusca), an intermediate host snail of Fasciola spp. PLoS One 2012, 7:e42172.

40. Ronquist F, Teslenko M, van der Mark P, Ayres DL, Darling A, Höhna S, Larget B, Liu L, Suchard MA, Huelsenbeck JP: MrBayes 3.2: efficient Bayesian phylogenetic inference and model choice across a large model space. Syst Biol 2012, 61:539-542.

41. Page RD: TREEVIEW: an application to display phylogenetic trees on personal computers. Comput Appl Biosci 1996, 12:357-358.

42. Peng $M$, Ichinomiya $M$, Ohtori M, Ichikawa $M$, Shibahara T, Itagaki T: Molecular characterization of Fasciola hepatica, Fasciola gigantica, and aspermic Fasciola sp. in China based on nuclear and mitochondrial DNA. Parasitol Res 2009, 105:809-815.

43. Mas-Coma S, Valero MA, Bargues MD: Chapter 2. Fasciola, lymnaeids and human fascioliasis, with a global overview on disease transmission, epidemiology, evolutionary genetics, molecular epidemiology and control. Adv Parasitol 2009, 69:41-146.

44. Masaoka T, Okamoto H, Araki K, Nagoya H, Fujiwara A, Kobayashi T: Identification of the hybrid between Oryzias latipes and Oryzias curvinotus using nuclear genes and mitochondrial gene region. Mar Genomics 2012, 7:37-41.

45. Gasser RB: Mutation scanning methods for the analysis of parasite genes. Int J Parasitol 1997, 27:1449-1463.

46. Reblánová M, Spakulová M, Orosová M, Králová-Hromadová I, Bazsalovicsová E, Rajský D: A comparative study of karyotypes and chromosomal location of rDNA genes in important liver flukes Fasciola hepatica and Fascioloides magna (Trematoda: Fasciolidae). Parasitol Res 2011, 109:1021-1028.

47. Srimuzipo P, Komalamisra C, Choochote W, Jitpakdi A, Vanichthanakorn P, Keha P, Riyong D, Sukontasan K, Komalamisra N, Sukontasan K, Tippawangkosol P: Comparative morphometry, morphology of egg and adult surface topography under light and scanning electron microscopies, and metaphase karyotype among three size-races of Fasciola gigantica in Thailand. Southeast Asian J Trop Med Public Health 2000, 31:366-373.

48. Pagès JR, Southgate VR, Tchuem Tchuenté LA, Jourdane J: Experimental evidence of hybrid breakdown between the two geographical strains of Schistosoma intercalatum. Parasitology 2002, 124:169-175.

49. Webster BL, Diaw OT, Seye MM, Webster JP, Rollinson D: Introgressive hybridization of Schistosoma haematobium group species in Senegal: species barrier break down between ruminant and human schistosomes. PLoS Negl Trop Dis 2013, 7:e2110.

50. Moné H, Minguez S, Ibikounlé M, Allienne JF, Massougbodji A, Mouahid G: Natural Interactions between $\mathrm{S}$. haematobium and $\mathrm{S}$. guineensis in the Republic of Benin. Sci World J 2012, 2012:793420.

51. Anderson TJ: The dangers of using single locus markers in parasite epidemiology: Ascaris as a case study. Trends Parasitol 2001, 17:183-188. 
52. Peng W, Yuan K, Zhou X, Hu M, Abs EL-Osta YG, Gasser RB: Molecular epidemiological investigation of Ascaris genotypes in China based on single-strand conformation polymorphism analysis of ribosomal DNA. Electrophoresis 2003, 24:2308-2315.

53. Itagaki T, Ichinomiya M, Fukuda K, Fusyuku S, Carmona C: Hybridization experiments indicate incomplete reproductive isolating mechanism between Fasciola hepatica and Fasciola gigantica. Parasitology 2011, 138:1278-1284.

doi:10.1186/1756-3305-7-150

Cite this article as: Liu et al:: Complete mitochondrial genomes of the

'intermediate form' of Fasciola and Fasciola gigantica, and their

comparison with $F$. hepatica. Parasites \& Vectors 2014 7:150.

\section{Submit your next manuscript to BioMed Central and take full advantage of:}

- Convenient online submission

- Thorough peer review

- No space constraints or color figure charges

- Immediate publication on acceptance

- Inclusion in PubMed, CAS, Scopus and Google Scholar

- Research which is freely available for redistribution 